\title{
Photonic Binding in Silicon-Colloid Microcavities
}

\author{
E. Xifré-Pérez, ${ }^{1,2}$ F. J. García de Abajo, ${ }^{3, *}$ R. Fenollosa, ${ }^{1,2}$ and F. Meseguer ${ }^{1,2, \dagger}$ \\ ${ }^{1}$ Centro de Tecnologías Físicas, Unidad Asociada ICMM-CSIC/UPV, Universidad Politécnica de Valencia, \\ Av. de los Naranjos s/n. 46022 Valencia, Spain \\ ${ }^{2}$ Instituto de Ciencia de Materiales de Madrid (CSIC), C/ Sor Juana Inés de la Cruz, 3, 28049 Madrid, Spain \\ ${ }^{3}$ Instituto de Óptica - CSIC, Serrano 121, 28006 Madrid, Spain \\ (Received 30 April 2009; revised manuscript received 21 July 2009; published 2 September 2009)
}

\begin{abstract}
Photonic binding between two identical silicon-colloid-based microcavities is studied by using a generalized multipolar expansion. In contrast with previous works, we focus on low-order cavity modes that resemble low-energy electronic orbitals. For conservative light intensities, the interaction between cavity modes with moderate $Q$ factors produces extremely large particle acceleration values. Optical forces dominate over van der Waals, gravity, and Brownian motion, and they show a binding-antibinding behavior in analogy to electronic binding. As these photonic forces are associated with relatively broad Mie mode resonances and they are not strongly influenced by sample absorption, our study opens a plausible avenue towards manipulation of high-refractive-index colloidal assemblies.
\end{abstract}

DOI: 10.1103/PhysRevLett.103.103902

PACS numbers: 42.60.Da, 78.20.Bh, 78.67.-n

The essence of electronic binding lies in the electromagnetic force resulting from the exchange of virtual photons. This constitutes the basis of chemistry. Similar effects appear in neighboring particles when they are irradiated by an intense light beam. But now, the interaction originates in the exchange of real photons. Photonic forces between nanophotonic systems have been studied in photonic crystals [1] and demonstrated for metallic nanoparticles $[2,3]$. These forces have been measured in high- $Q$-factor microcavities with $\delta$-like resonant modes [4]. One of the simplest microcavities is a dielectric microsphere $[5,6]$. However, typical dielectric spheres made of silica or polystyrene can only sustain large modal-number modes because of their low refractive-index contrast relative to air or water environments [7]. As these modes are mostly localized in the surface of the cavity, they would be equivalent to atoms excited into high-energy orbitals. Typically, small particle sizes [1] or optical absorption in metals [2] limit the magnitude of photonic forces, which are then difficult to push well above other competing factors such as van der Waals attraction and Brownian motion. A promising route to overcome this problem relies on the use of high-refractive-index particles. Recently, Fenollosa et al. [8] have reported on the synthesis of silicon colloids with spherical shape and very smooth surface. Because of its high refractive index, silicon colloids are excellent optical microcavities, and they show well-defined, low-order Mie modes in the near-IR, the spatial distributions of which bear strong resemblance to those of low-energy atomic orbitals. Therefore, silicon colloids can be thought of as a typical textbook prototype of photonic atoms for studying light-induced binding.

In this work, we study the electromagnetic interaction between neighboring silicon spheres by analyzing lightdriven photonic forces as a function of both optical wavelength and interparticle distance. In contrast to previous works, we investigate photonic forces mediated by loworder modes of small index $(n, m)$. Because of both the high refractive index of silicon and the small volume of the cavities, these photonic forces give rise to particle accelerations that are much stronger (up to 2 orders of magnitude) than those calculated for low-refractive-index particles $[7,9,10]$. Additionally, as these forces originate in relatively broad resonances, they are less influenced by material absorption and can be more easily implemented in experiments. Finally, we argue that particle motion driven by these forces and by other factors such as stochastic Brownian forces occurs on a long time scale compared to the lifetime of the particle optical resonances, and therefore, optical forces can be safely studied as in molecular chemistry assuming static particles (Born-Oppenheimer approximation).

Different theoretical approaches have been elaborated to calculate Mie modes and interparticle forces produced by external electromagnetic fields $[11,12]$. Here, we use the semi-analytical method based upon multiple elastic scattering of multipole expansions (MESME) [12], which provides a rigorous solution of Maxwell's equations for arbitrary distributions of spheres. We have accounted for multipoles up to an orbital number $l=14$ in order to achieve converged results. We focus here on dimers formed by two silicon spheres of radius $r=750 \mathrm{~nm}$, irradiated by a light plane wave in the $\lambda=2.3-3.3 \mu \mathrm{m}$ wavelength range (far away from the absorption edge), in which the permittivity $\epsilon$ varies by just $1 \%$ [13], so that we can safely assume a real constant value of $\epsilon=12$. (We have verified that a small imaginary part in $\epsilon$ has no significant effect, see [14]). The dimer spheres are taken to be very close to each other, with a surface-to-surface separation $S \ll r$.

We start by inspecting the scattering cross section of the dimer $\sigma$, as obtained from MESME [12]. Figure 1 shows a contour plot of $\sigma$ normalized per sphere as a function of the 
size parameter $\alpha=2 \pi r / \lambda$. The dimer axis is oriented perpendicularly with respect to the light wave vector $k$ (see insets of Fig. 1). We consider two different light polarizations ( $p$ and $s$, see Fig. 1). Both polarizations can excite $a_{m n}$ and $b_{m n}$ modes that correspond to transversemagnetic and transverse-electric symmetry, respectively. Peaks in $\sigma$ for the dimer are labeled $a_{m n}^{\prime}$ and $b_{m n}^{\prime}$, as they seem to originate in the corresponding $a_{m n}$ and $b_{m n}$ modes of the single sphere [15]. From the contour plot, substantial differences between both polarizations can be observed. For $p$ polarization [Fig. 1(a)], as expected, $a$ modes from neighboring particles couple because they are coplanar and they shift to lower size-parameter values when the spheres approach each other. Also, $b$ modes have weaker mutual interaction [see lower inset of Fig. 1(a)]. In contrast, $s$ polarization entails larger $b$-mode interaction, and therefore stronger optical forces, since they are coplanar [see lower inset of Fig. 1(b)]. They also shift to lower size parameter as the spheres approach each other. For $s$ polarization, some branches coming from $b_{31}$ and $b_{41}$ singlesphere modes shift to smaller $\alpha$ 's at small separations. However, these results cannot be in general accounted for by the naïve picture predicting dimer interaction for modes whose orbits are coplanar (see insets of Fig. 1). For $p$ polarization, the $b_{41}^{\prime}$ resonance splits into several modes
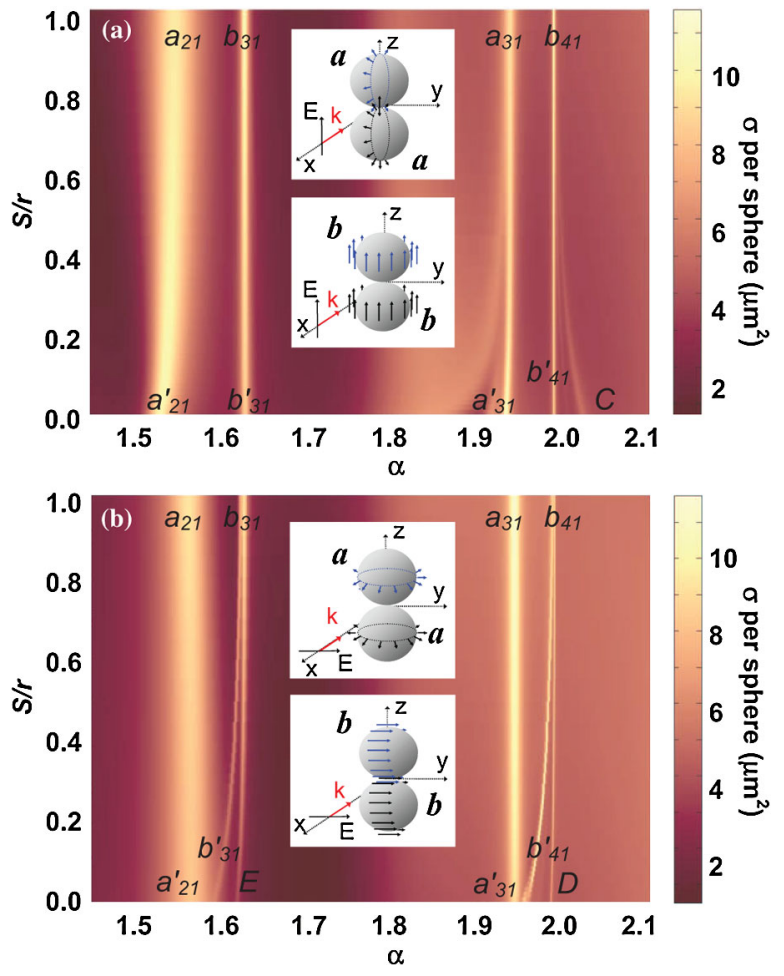

FIG. 1 (color online). Contour plot of the scattering cross section $\sigma$ per sphere as a function of the size parameter $\alpha=$ $2 \pi r / \lambda$ and the distance between the spheres for (a) $p$ polarization and (b) $s$ polarization. Insets: Schematic representation of the dielectric-sphere dimer under consideration, along with the $a$ and $b$ Mie modes of the individual particles. The color scale is given for particles of radius $r=750 \mathrm{~nm}$. (several branches in the contour plot). One of them $\left(b_{41}^{\prime}\right)$ does not shift, as expected, but the other ( $C$ branch) shifts upwards, and simultaneously, the $a_{31}^{\prime}$ branch shifts downwards. As we show later, this can be related to a bindingantibinding behavior of a photonic molecule.

The upper curves in Figs. 2(a) and 3(a) show the acceleration produced in each dimer particle by optical forces, normalized to the acceleration of gravity, $a / g$, for $r=$ $750 \mathrm{~nm}$ and $S / r=0.05(S=37.5 \mathrm{~nm})$, assuming a moderate light intensity of $10^{-4} \mathrm{~W} / \mu \mathrm{m}^{2}$. The magnitude of $a / g$ and the width of the force peaks $(>1 \mathrm{~nm})$ are both in a range that is experimentally accessible for the light wavelengths under consideration. Additionally, these photonic forces are less sensitive to material absorption (see [14]) than those reported for low-refractive-index cavities [7].

It is instructive to discuss first the scattering cross section in order to understand the dimer interaction. We show $\sigma$ in the lower curves of Figs. 2(a) and 3(a) for the dimer (thick curves) and for a single sphere (thin curves). The dimer spectra for both $p$ and $s$ polarization exhibit signifi-

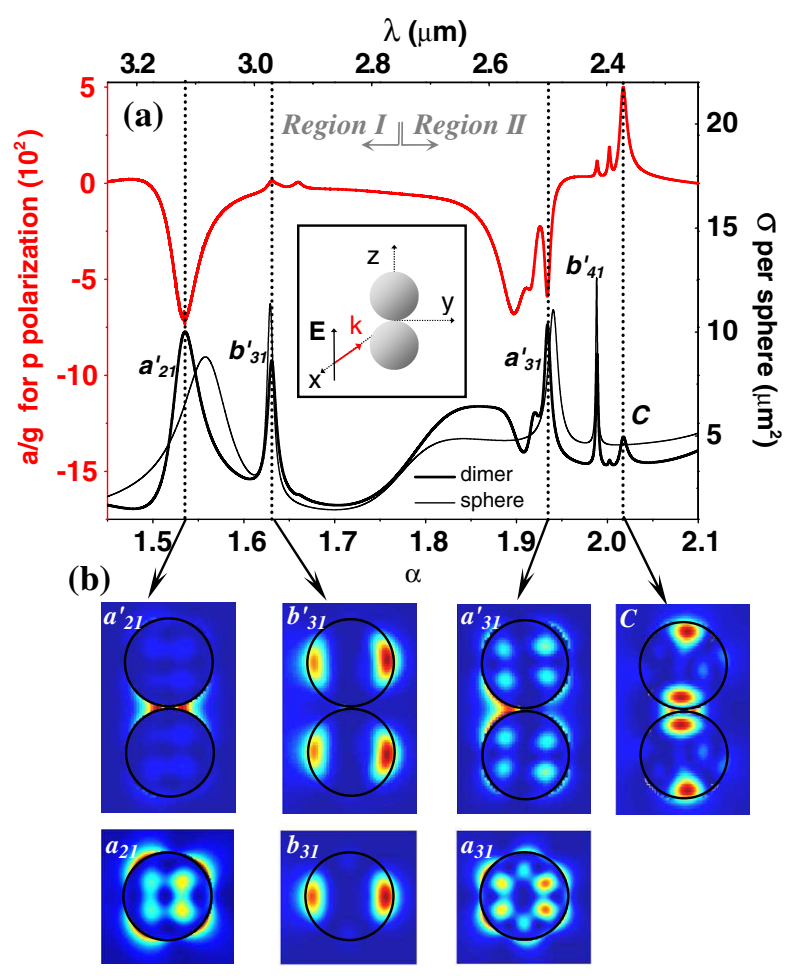

FIG. 2 (color online). (a) Scattering cross section of the dimer (right scale and thick black/lower curve) and photonic-forceinduced acceleration (in units of the acceleration of gravity $g$ ) along the dimer-axis $z$ direction (left scale and red/upper curve) for $p$ polarization as a function of size parameter $\alpha=2 \pi r / \lambda$ (bottom axis) and wavelength $\lambda$ (top axis). The scattering cross section for an individual sphere is also plotted (thin black/lower curve). The spheres radius is $r=750 \mathrm{~nm}$ and the surface-tosurface distance is $S=r / 20$. The inset shows the scattering configuration. (b) Electric-field intensity distribution in the $x-z$ plane for some of the dimer modes (upper panels), and the corresponding single-sphere modes (lower panels). 


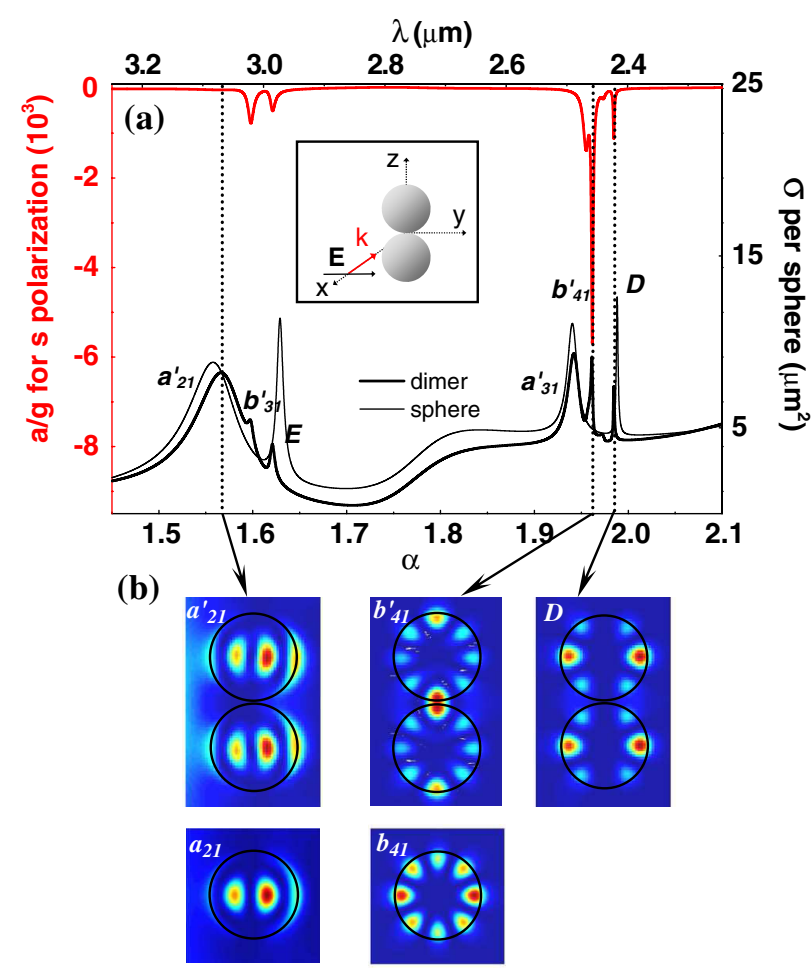

FIG. 3 (color online). Same as Fig. 2 for $s$ polarization (i.e., incoming electric field perpendicular to dimer axis).

cant differences with respect to single-sphere scattering, as the interparticle interaction can change completely the field distribution (see below). In particular, $\sigma$ displays strong modifications in the dimer compared to the individual particle in the spectral range near $a$ modes for $p$ polarization [Fig. 2(a)], and even new peaks show up. However, $b$ modes are much less affected by interparticle interaction. In contrast, the opposite is true for $s$ polarization [see Fig. 3(a)], since $a$ modes are hardly affected and $b$ modes split into several peaks with resonant frequencies differing from those of the single sphere. All these features have consequences on the photonic force shown in the upper curves of Figs. 2(a) and 3(a). Positive and negative values of $a / g$ are observed, indicating that the interaction can be repulsive or attractive, respectively. For $p$ polarization, Fig. 2 shows attractive and repulsive forces that depend on the wavelength. Two different spectral regions can be distinguished: region I $(\alpha<1.75)$ shows $a_{21}^{\prime}-b_{31}^{\prime}$ modes and region II $(\alpha>1.75)$ is dominated by $a_{31}^{\prime}-b_{41}^{\prime}$ modes. The binding-antibinding interplay is more easily discernable in region II, where attractive (binding) forces are associated to Mie resonances that shift to lower light frequency for decreasing interparticle distance (see $a_{31}^{\prime}$ in Fig. 1). In contrast, repulsive (antibinding) forces are associated to resonances shifting to larger frequencies (see $C$ branch near the $b_{41}$ resonance in Fig. 1). This was not expected from the naïve picture that $p$ polarization does not excite $b_{n m}$ modes. This rich, unexpected bindingantibinding interplay bears some resemblance to the situation encountered in atomic binding.
In order to better understand the origin of photonic binding, we have plotted the spatial distribution of the electric near-field intensity along the $x-z$ plane $(y=0)$ for selected values of the size parameter corresponding to the maxima of $\sigma$ [Fig. 2(b)]. The intensity map of the single-particle $a_{21}$ mode is dramatically modified in the dimer, as the field leaks out to the interparticle region (see lower and upper panels corresponding to modes $a_{21}$ and $a_{21}^{\prime}$ of the single particle and dimer, respectively). However, the near-field of $b$ modes is similar in the dimer and in the individual sphere (see maps corresponding to modes $b_{31}$ and $b_{31}^{\prime}$ ). Region II of Fig. 2(a) shows complex binding and antibinding behavior, and the intensity maps are again helpful. We concentrate on the narrow dip and peak of the forces corresponding to the $a_{31}^{\prime}$ and $C$ resonances, respectively. A common feature in the intensity maps for both binding and antibinding regions is the strong modification of the field distribution with respect to a single sphere (see plots for modes $a_{31}$ and $a_{31}^{\prime}$ ). In previous studies for much larger particles [9], the variation of the fields along the sphere surfaces is similar to guided modes in a planar cavity, thus leading to simple rules that link the relative phase of the field in each sphere to the attractive or repulsive character of the resulting force. Unfortunately, this is not the case in our dimer, which is not much larger than the wavelength, thus involving the full complexity of the near field to elucidate the sign of the force. Whether a simple criterium can be still formulated in these small cavities remains an open question to us.

Focusing now on $s$ polarization (Fig. 3), we first notice that only attractive forces appear, although antibinding forces are possible for other scattering configurations [7]. This is consistent with the results of Fig. 1(b) showing that only $b$ resonances move downwards as the particles come closer. To get further insight into these photonic forces, we have represented near-field maps corresponding to the noninteracting mode $a_{21}^{\prime}$ and the two binding modes $b_{41}^{\prime}$ and $D$ in Fig. 3(b), as compared to the modes of an individual sphere. Mode $D$ does not exist in the single particle. Figure 3(b) shows that the field distribution and frequency of the single-sphere mode $a_{21}$ are nearly unchanged by the presence of the twin sphere. The performance of mode $b_{41}$ is however different: $\sigma$ is significantly modified, and, although the intensity maps for the dimer and the single sphere show the same number of intensity peaks per sphere, we can see that the electric field is dramatically shifted to the interparticle region, which results in a very strong attractive force.

Because of the high refractive index of silicon and the low volume of the cavity, the particles are subject to very high accelerations (see [14]), up to 2 orders of magnitude higher than those reported for either low-refractive-index, large size microcavities [9], or low-refractive-index, small size particles [7]). The most salient feature of our silicon dimer is the very nature of the Mie resonances, which are intense even for low modal numbers $(n, m)$ and have a 


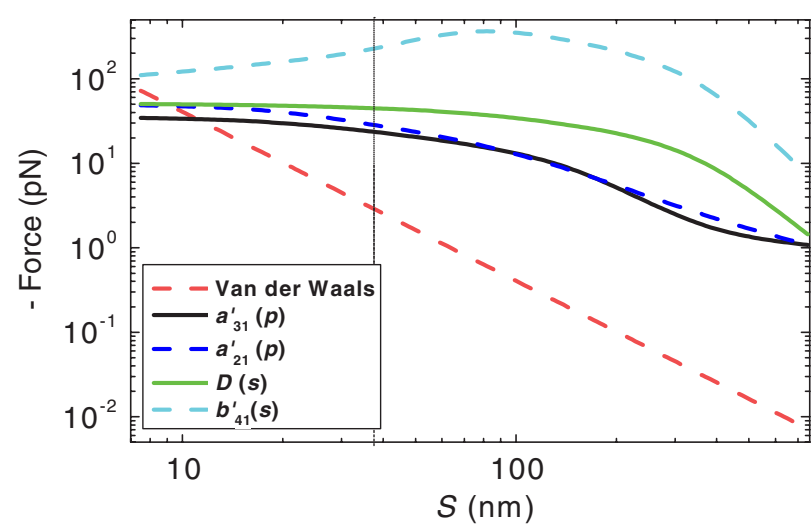

FIG. 4 (color online). Attractive photonic force in the dimer for different modes and light polarizations ( $p$ and $s$ ) as a function of the separation $S$ between the spheres. The photonic force for a light intensity of $10^{-4} \mathrm{~W} / \mu \mathrm{m}^{2}$ is compared to the van der Waals force. The vertical dashed line corresponds to a distance $S=$ $r / 20$.

natural linewidth much broader than those of large-size silica-made cavities. As a result, the silicon dimer is more robust against the effect of optical absorption, as shown in [14]. Additionally, our calculated resonance linewidths are of the order of several nanometers, which makes experiments more feasible than those based upon high- $Q$-factor modes previously discussed [7,9].

Actual experiments are sensitive to other types of forces, such as van der Waals (vdW), and Brownian motion, so we compare their relative importance in what follows. In particular, the vdW force can be approximated as [16]

$$
F_{\mathrm{vdW}}=\frac{A r}{12 S^{2}},
$$

where $A$ is the Hamaker constant $\left(A=6.5 \times 10^{-20} \mathrm{~J}\right.$ for silicon) [17]. Figure 4 shows both vdW forces (lower dashed curve) as well as the attractive forces corresponding to several resonances as a function of $S$ for a light intensity of $10^{-4} \mathrm{~W} / \mu \mathrm{m}^{2}$. In all cases, $\mathrm{vdW}$ forces are significantly weaker than optical forces (up to 3 orders of magnitude). Only for very small separations $S<10 \mathrm{~nm}$ do vdW forces overcome optical binding. Finally, Brownian forces $(F=$ $K T / r, K T$ being the thermal energy) are negligible compared to optical forces.

We have so far assumed the particles to be at rest during the lifetime of the optical cavity modes setting up the optical forces (similar to the Born-Oppenheimer approximation in quantum chemistry). This approximation is only justified if the mechanical motion of the particles is negligible during the time needed to build up the electromagnetic field of the cavity modes. In particular, for the $b_{41}^{\prime}$ resonance [see Fig. 3(a)] with a $Q \approx 10^{3}$, the field takes around 10 picoseconds to build up, much quicker than other temporal processes such as Brownian forces (of the order of milliseconds at room temperature) or optically driven motion.
In conclusion, resonant optical modes of dielectricsphere dimers have been analyzed in the near-infrared region. We find a binding-antibinding interplay similar to atomic binding. Attractive-repulsive forces are consistent with up-down frequency shifts of the modes as the particles are brought close to each other. The large permittivity of silicon allows us to obtain intense optical forces with moderate light intensities mediated by low-order Mie resonances and with relatively broad profiles that make experimental detection feasible. We have shown that photonic forces are much stronger than van der Waals and Brownian forces. We expect that the relative position of the spheres in the silicon dimer can be finely controlled using illumination with appropriate wavelength and polarization. We hope that our work will open a new avenue towards photonic binding relying on silicon colloids in the nearinfrared region.

This work has been partially supported by the Spanish CICyT (Projects MAT2006-03097, MAT2007-66050, TEC2006-06531, and Consolider CSD2007-046) and by the EU (NMP4-SL-2008-213669-ENSEMBLE).

*To whom correspondence should be addressed: jga@cfmac.csic.es

${ }^{\dagger}$ To whom correspondence should be addressed: fmese@ fis.upv.es

[1] M. I. Antonoyiannakis and J. B. Pendry, Europhys. Lett. 40, 613 (1997).

[2] A. Hallock, P. Redmon, and L. Brus, Proc. Natl. Acad. Sci. U.S.A. 102, 1280 (2005).

[3] M. Righini et al., Nature Phys. 3, 477 (2007).

[4] M. Eichenfield et al., Nat. Photon. 1, 416 (2007).

[5] P. R. Conwell, P. W. Barber, and C. K. Rushforth, J. Opt. Soc. Am. A 1, 62 (1984).

[6] D. W. Vernooy et al., Opt. Lett. 23, 247 (1998).

[7] J. Ng et al., Opt. Lett. 30, 1956 (2005).

[8] R. Fenollosa, F. Meseguer, and M. Tymczenko, Adv. Mater. 20, 95 (2008).

[9] M. L. Povinelli et al., Opt. Express 13, 8286 (2005).

[10] S. Mohanty, J. Andrews, and P. Gupta, Opt. Express 12, 2746 (2004).

[11] C. Bohren and D. Huffman, Absorption and Scattering of Light by Small Particles (John Wiley \& Sons, New York, 1998).

[12] F. J. García de Abajo, Phys. Rev. Lett. 82, 2776 (1999); Phys. Rev. B 60, 6086 (1999).

[13] E. Palik, Handbook of Optical Constants of Solids (Academic Press, New York, 1985), Vol. 1.

[14] See EPAPS Document No. E-PRLTAO-103-002938. For more information on EPAPS, see http://www.aip.org/ pubservs/epaps.html.

[15] T. Mukaiyama et al., Phys. Rev. Lett. 82, 4623 (1999).

[16] J. Israelachvili, Intermolecular and Surface Forces (Academic Press, London, 1985).

[17] C. Brinker and G. Scherer, Sol-Gel Science: The Physics and Chemistry of Sol-Gel Processing (Academic Press, London, 1990). 